(Aus dem physiologischen Institut der Universität Jena.)

\title{
Ultramikroskopische Beobachtungen an Muskel- und Geisselzellen.
}

\author{
Von
}

\section{Hans Stübel.}

Eine Reihe physiologischer und physikalisch-chemiseher Erscheinungen haben $\mathrm{Höber}{ }^{1}$ ) dahin geführt, die Hypothese auszusprechen, dass im Erregungsvorgang als Komponente ein Kolloidvorgang, eine Veränderung des kolloidalen Zustandes der erregbaren Substanz, enthalten ist. Auf Grund dieser Hypothese hat Hö ber ${ }^{2}$ ) versucht, direkt einen derartigen Kolloidvorgang im Nerven zu beobachten und zwar mit Hilfe der Dunkelfeldbeleuchtung oder, besser gesagt, der Ultramikroskopie. Er beobachtete zu diesem Zwecke verschiedene Nerven (N. ischiadicus des Frosches, N. olfactorius des Hechtes) bei Beleuchtung mit dem Paraboloidkondensor, während sie mit dem Induktionsstrom gereizt wurden. Früher hatte bereits L. Auerbach ${ }^{3}$ ) den markhaltigen Nerven mit Hilfe des Ultramikroskopes untersucht. Höb er konnte nur über negative Versuchsergebnisse berichten. Der markhaltige Nerv scheint für eine derartige Untersuchung auch nicht gerade geeignet zu sein. Einmal sind die markhaltigen Nervenfasern verhältnismässig dick und schon daher der ultramikroskopischen Methode schwer zugänglich, und ausserdem werden noch durch die stark lichtbrechende Markscheide ungünstige Beobachtungsbedingungen geschaffen. Dazu kommt, dass es immerhin mit Schwierigkeiten verbunden ist, einen Nerven so zu zerzupfen, dass er einerseits der ultramikroskopischen Beobachtung zugänglich

1) Höber, Die physikalisch-chemischen Vorgänge bei der Erregung. Zeitschr. f. allgem. Physiol. Bd. 10 S. 173. 1910.

2) Höber, Untersuchung erregbarer Nerven bei Dunkelfeldbeleuchtung: Pflüger's Arch. Bd. 133 S. 254. 1910. - Höber, Physikalische Chemie der Zelle und der Gewebe, 2. Aufl., S. 419.1911.

3) L. Auerbach, Neurol. Zentralbl. 1908 S. 994 . Pflüger's Arch. Bd. 143 S. 574.1911. 
ist, andererseits aber seine normale Funktionsfähigkeit behält. Ungünstig scheinen mir für den markhaltigen Nerven die Verhältnisse auch deshalb zu liegen, weil ja hier der Erregungsvorgang mit einer ausserordentlich grossen Geschwindigkeit abläuft. Aus diesem Grunde ist es sehr wohl denkbar, dass eine während der Erregung auftretende Veränderung des Brechungsexponenten, auch wenn sie vorhanden wäre, sich subjektiv überhaupt nicht an diesem Objekte beobachten lässt. Ebensowenig wie der markhaltige Nerv ist nach Höber der marklose Hechtolfactorius zur Beobachtung mit dem Ultramikroskop geeignet. Dieselbe Erfahrung habe ich mit den Nervenfasern des Bauchstranges und seiner Seitenäste vom Blutegel gemacht. Hier ist eine fibrilläre Struktur bei Dunkelfeldbeleuchtung nicht oder nur andeutungsweise zu sehen; sie wird vielmehr durch unzählige, stark lichtbrechende Körnchen fast völlig verdeckt.

Bei der Bedeutung derartiger Untersuchungen für unsere Auffassung von der Natur des Erregungsvorganges erschien es mir trotz dieser negativen Ergebnisse von Interesse, abgesehen vom Nerven auch andere erregbare Gewebselemente mit Hilfe des Ultramikroskopes während ihrer Tätigkeit zu beobachten. Auch hier hatte die Untersuchung stets ein negatives Resultat in dem Sinne, dass niemals eine durch den Ablauf des Erregungsvorganges bedingte Helligkeitsveränderung bei Dunkelfeldbeleuchtung wahrgenommen werden konnte. Ich will daher hier nur ganz kurz über meine ultramikroskopischen Beobachtungen an glatten Muskelzellen, an den Myoidfäden von Infusorien sowie an Geisselund Flimmerzellen berichten.

Glatte Muskelzellen schienen mir aus verschiedenen Gründen geeignete Objekte zu sein. Einmal ist hier der Erregungsablauf ein sehr langsamer, und es ist verhältnismässig einfach, geeignete und ausdauernde Präparate herzustellen. Vor allem aber lässt sich an manchen glattmuskeligen Organen eine Erscheinung feststellen, die geradezu darauf hinweist, dass hier der Kontraktionsvorgang mit der Ausfällung eines Kolloides einhergeht, indem nämlich die jeweils kontrahierte Muskelpartie ein trübes, weissliches oder bläuliches Aussehen erhält. Über derartige Erscheinungen hat Bieder$\operatorname{man} n^{1}$ ) bereits früher Beobachtungen angestellt und hat dabei an

1) Biedermann, Studien zur vergleichenden Physiologie der peristaltischen Bewegungen. II. Die lokomotorischen Wellen der Schneckensohle. Pflüger's Arch. Bd. 107 S. 15.1905. 
die Möglichkeit einer Kolloidfällung gedacht. So sind z. B. die peristaltischen Bewegungen der Schneckensohle (Helix) besonders deswegen gut zu verfolgen, weil hier mit dem Fortschreiten einer Kontraktionswelle stets eine Trübung und weissliche Verfärbung der kontrahierten Partie des Sohlenmuskels einhergeht. Bleibt eine derartige Kontraktionswelle stehen, $d$. h. verharrt eine Muskelpartie in Dauerkontraktion, so behält diese Stelle die weissliche Verfärbung bis zum Wiedereintritt der Erschlaffung bei. Besonders schön lässt sich diese Trübung und Verfärbung an den glatten Muskeln mancher niederen Meerestiere beobachten, z. B. an den Laternenmuskeln des Seeigels. Diese Muskeln sind in erschlafftem Zustande fast glashell und durchsichtig; kontrahierte Stellen dagegen sind undurchsichtig und haben eine blauweisse Färbung.

Wenn auch bei der Beobachtung dieser Erscheinungen der Gedanke der nächstliegende ist, dass hierbei die Ausfällung eines kolloidalen Körpers vonstatten geht, so darf doch nicht unerwähnt bleiben, dass auch eine andere Deutung dieser Trübungen möglich ist, worauf erst in jüngster Zeit W. Pauli ${ }^{1}$ ) aufmerksam gemacht hat. Nach Pauli können nämlich ganz analoge Erscheinungen durch reversible Wasserverschiebungen in Leimgallerten entstehen. Ebenso könnten nach $\mathrm{Pauli}$ auch „reversible Wasserverschiebungen zwischen Muskelplasma und Fibrille" zu Brechungsunterschieden führen. Die Dentung der Trübung in diesem Sinne lässt sich in Einklang mit der von $\mathrm{Pauli}$ aufgestellten Kontraktionstheorie bringen. Mag man jedoch die beschriebenen Erscheinungen auffassen wie man will, so bleibt ein Studium derselben mit dem Ultramikroskop doch von Interesse.

Bei den ultramikroskopischen Beobachtungen glattmuskeliger Organe bediente ich mich des Siedentopf'schen Paraboloidkondensors. Dieser wird zwar von dem Kardioidkondensor, den wir gleichfalls Siedent opf verdanken, an Lichtstärke noch übertroffen, ist aber für unsere Zwecke geeigneter, da er eine grössere Sehtiefe und ein weiteres Gesichtsfeld ermöglicht. Als Untersuchungsobjekt diente mir zuerst die Harnblase des Frosches, welche sich so dünn ausspannen lässt, dass sie bequem bei Dunkel-

1) W. Pauli, Kolloidchemie der Muskelkontraktion. Über den Zusammenhang von elektrischen, mechanischen und chemischen Vorgängen im Muskel. Dresden und Leipzig 1912. 
feldbeleuchtung zu betrachten ist. Ein derartiges Präparat gewährt bei Dunkelfeldbeleuchtung eine vorzügliche Übersicht über sämtliche Gewebsbestandteile der Harnblase, die Bündel glatter Muskelzellen, Blutgefässe, dickere Nervenstämme und Epithelzellen, so dass es sich auch zu Demonstrationszwecken gut ejgnen dürfte. Abgesehen davon, dass es schwierig und mehr oder weniger vom Zufalle abhängig ist, durch irgendwelche Reize an diesem Präparate die Muskulatur zur Kontraktion zu bringen, ohne gleichzeitig die Exaktheit der Dunkelfeldbeleuchtung zu beeinträchtigen, ist die Beobachtung einer in den Muskelzellen während der Kontraktion eventuell auftretenden Helligkeitsveränderung deswegen erschwert, weil hier auch im erschlafften Zustande die einzelnen Muskelzellen im Dunkelfeld nicht optisch leer sind, sondern ziemlich helleuchtend erscheinen.

Diese letztere Schwierigkeit machte sich auch bei dem zweiten Objekt, welches mir zur Untersuchung diente, dem Musculus retractor penis der Weinbergschnecke (Helix pomatia), geltend. Dieser Muskel erschien mir deswegen gut geeignet, weil er erstens parallelfaserig ist und zweitens, weil er sich ganz ausserordentlich lang ausdehnen lässt, wobei er sehr dünn wird. Um den Muskel in gedehntem Zustande zu fixieren, wird er am besten mit seinen beiden Ansatzpunkten (Schale und Penis) mit Hilfe von Deckglaskitt auf dem trockenen Objektträger festgeklebt, während der Bauch des Muskels in Schneckenblut unter dem Deckglase liegt. Selbstverständlich darf aber ein derartiges Präparat nicht maximal gedehnt sein, wenn man an ihm noch Kontraktionserscheinungen wahrnehmen will. Um eine einzelne Stelle des Muskels zur Kontraktion zu bringen, durchströmt man den Muskel mit dem konstanten Strom. Man erhält dann an der Kathode eine Dauerkontraktion, wie das Biedermann ${ }^{1}$ ) früher an verschieden glattmuskeligen Organen festgestellt hat. Aber auch an diesem Muskel stellten sich ähnliche Schwierigkeiten wie an der Muskulatur der Harnblase heraus: Die Muskelzellen sind auch im Ruhezustande nicht optisch leer, sondern trübe und noch dazu mit stark lichtbrechenden Körnchen erfültt. Daher wären an diesem Präparate nur sehr erhebliche Heiligkeitsunterschiede $\mathrm{z}$ wischen kontrahierten und erschlafften Partien bemerkbar geworden; es waren jedoch überhaupt keine derartigen Unterschiede zu sehen.

1) Biedermann, Zur Physiologie der glatten Muskeln. Pflüger's Arch. Bd. 46 S. 398. 1889. 
Das günstigste glattmuskelige Organ, welches ich benutzte, waren die Laternenmuskeln der Seeigel, und zwar nahm ich die Muskeln von kleineren Exemplaren von Strongylocentrotus lividus (aus Triest, Schalendurchmesser bis $5 \mathrm{~cm}$ ). Am besten eigneten sich die Schliessmuskeln der Zähne ${ }^{1}$ ). Der Muskel wurde mit den beiden Knochenstücken, an denen er ansetzt, herausgenommen, die Knochenstücke wurden auf dem Objektträger mit Deckglaskitt fixiert, und der in Seewasser liegende Muskel wurde mit einem Deckglassplitter bedeckt. Da der Muskel sofort in einen tonischen Kontraktionszustand gerät, wenn er entspannt wird, muss man ihn bereits während der Präparation durch vorsichtiges Anhängen eines kleinen Gewichtes ausdehnen. An die Knochenstücke wurden dann unpolarisierbare Pinselelektroden angelegt und der Muskel mit konstantem Strom durchströmt. Die Muskelzellen der Laternenmuskeln haben den Vorzug, dass sie bei Dunkelfeldbeleuchtung optisch fast leer sind und infolgedessen hellkonturiert, aber im Inneren dunkel erscheiuen. Jedoch liess sich auch hier an einer kontrahierten Stelle (Kathode) keine Veränderung der optischen Eigenschaften des Zellinhaltes erkennen.

Ein viel günstigeres Objekt zum Studium des Kontraktionsvorganges bei Dunkelfeldbeleuchtung als die glatte Muskelzelle ist der Stielmuskel der Vorticellen. Sowohl die Scheide des Stielmuskels als dieser selbst sind optisch fast vollkommen leer, nur auf dem Muskelfaden und in demselben zeigen sich ganz vereinzelt und in unregelmässigen Abständen runde, leuchtende Körper, welche die Beobachtung in keiner Weise stören. Während der Kontraktion des Stielmuskels lässt sich niemals eine Veränderung in seinem: Inneren beobachten. Eine Aufhellung könnte höchstens einmal durch eine Übereinanderlagerung einzelner Teile des spiralig aufgewundenen Stieles vorgetäuscht werden.

Während also bei allen bis jetzt angeführten Objekten niemals eine Veränderung des Brechungsexponenten der erregbaren Substanz während ihrer Tätigkeit nachweisbar war, liess sich dies bei der Beobachtung mancher Geissel- und Flimmerzellen viel schwerer entscheiden. Da man Geissel- und Flimmerzellen auch noch in sehr dünner Schicht $(2-4 \mu)$ zwischen Objektträger und

1) Vgl. Lang, Lehrbuch der vergleichenden Anatomie der wirbellosen Tiere S. 992. Jena 1894. 
Deckglas bringen kann, und da es sich hier um die Bewegung einfach gebauter, linearer Objekte handelt, so liegen die Bedingungen für die ultramikroskopische Untersuchung besonders günstig. Man kann mit Vorteil sowohl starke Lichtquellen als starke Vergrösserungen anwenden. Als Lichtquelle diente eine Handregulierbogenlampe, vor welche zur Absorption der Wärmestrahlen eine Küvette mit $1 / 2 \%$ iger Kupfersulfatlösung gestellt wurde. Ferner wurde zur Dunkelfeldbeleuchtung der Siedentopf'sche Kardioidkondensor angewendet, der bekanntlich die beste Strahlenvereinigung und somit auch die intensivste Beleuchtung ermöglicht. Der Kardioidkondensor hat für die Untersuchung lebender Objekte auch noch den Vorteil, dass er nur eine sehr kleine, punktförmige Stelle des Präparates beleuchtet, und diese Stelle allein wird der immerhin oft schädlichen Wirkung des intensiven Lichtes ausgesetzt, während das ganze übrige Präparat unversehrt bleibt. Als Objektiv wurde meist Zeiss' Apochromat, $3 \mathrm{~mm}$ Brennweite, als Okular dabei Kompensationsokular 12 (Vergrösserung 1000) oder Kompensationsokular 18 (Vergrösserung 1500) benutzt.

Zur Beobachtung der Geisselbewegung dienten mir hauptsächlich die Spermatozoen des Frosches (Rana esculenta, seltener R. temporaria), welche den Vorzug haben, dass hier die Geisselbewegung langsam und sehr regelmässig vonstatten geht. In einem Tröpfchen Hodensubstanz, welches in physiologischer Kochsalzlösung verteilt wird, finden sich neben vielen unbeweglichen auch zahlreiche bewegliche Spermatozoen, so dass in einem solchen Präparat stets reichlich Untersuchungsmaterial vorhanden ist. Am meisten eignen sich solche Spermatozoen zur Beobachtung, deren Kopf ruhig liegt, während die Geissel sich noch bewegt und das Spermatozoon also keine Ortsbewegung mehr ausführt. Der Kopf des Spermatozoons ist optisch leer; sein ultramikroskopisches Bild entspricht also dem der meisten Zellkerne. Der Geisselfaden zeigt sich in Ruhe als ein gleichmässig beller Lichtstreifen, beim Frosch ohne doppelte Kontur. An den Spermatozoen des Frosches sieht man nun unter diesen Umständen eine Erscheinung, die sich auf den ersten Blick in dem Sinve deuten liesse, dass mit der Bewegung der Geissel eine ultramikroskopisch sichtbare Veränderung der optischen Eigenschaften ihrer Substanz, nämlich eine Veränderung des Brechungsexponenten, einhergeht. Bei jeder Schwingung der Geissel wandert ein heller Lichtschein von dem Kopf des Spermatozoons aus über die ganze 
Geissel weg, in dem Sinne, dass die jeweils am meisten gekrümmte Strecke des Fadens den übrigen Faden an Helligkeit bedeutend übertrifft. Diese Lichterscheinung kann durch Ursachen von zweierlei Art bedingt sein: erstens kann die Erscheinung durch äussere Ursachen hervorgerufen werden, die durch die Art der Beleuchtung entstehen, und zweitens können, wie soeben angeführt, Veränderungen der kontraktilen Substanz des Geisselfadens, nämlich Veränderungen des Brechungsexponenten und mithin auch der physikalisch-chemischen Beschaffenheit, also innere Ursachen, in Betracht kommen.

Von dea äusseren, durch die Beleuchtung gegebenen Ursachen, die derartige Lichterscheinungen bedingen können, ist zuerst ein Fehler zu nemnen, der bei Dunkelfeldbeleuchtung sehr lejcht zu Täuschungen führen kann, nämlich eine nicht ganz genau allseitige Beleuchtung. Schon eine minimale Drehung des Spiegels genügt, um bei Anwendung des Kardioidkondensors die Beleuchtung völlig ungleichmässig zu machen, indem einerseits dann nur an bestimmten Stellen Licht abgebeugt wird, während andere Stellen desselben Objektes überhaupt nicht beleuchtet und damit nicht abgebildet werden können. Es ist daher unbedingt notwendig, sich stets vorerst mit Hilfe einer schwäcberen Vergrösserung zu vergewissern, dass die Beleuchtung vollkommen zentrisch ist. Um ganz sicher eine allseitige, gleichmässige Beleuchtung zu erhalten, ist es vorteilhaft, zwischen Spiegel und Kondensor noch eine blaue Mattglasscheibe einzuschalten, welche allerdings die Intensität der Beleuchtung herabsetzt. Es lässt sich nun auch bei vollkommen zentrischer Beobachtung und bei Zwischenschaltung einer Mattglasscheibe der erwähnte wandernde Lichtschein beobachten; jedoch kommt andererseits dieser Lichtschein bei nicht $\mathrm{ganz}$ zentrischer Beleuchtung unter Umständen stärker zum Ausdruck, was immerhin schon zu Bedenken Anlass gibt.

Auf eine weitere äussere Ursache, durch die die Veränderung der Helligkeit bei der Bewegung des Geisselfadens der Froschspermatozoen hervorgerufen werden könnte, hat neuerdings Si ed en to $\mathbf{f}^{1}$ ) hingewiesen. Siedent opf hat vor allem an Spirochäten ganz ähnliche Beobachtungen gemacht. Bei der Bewegung einer Spirochäte kann unter Umständen ein ebensolcher wandernder Licht-

1) Siedentopf, Über ultramikroskopische Abbildung linearer Objekte. Zeitschr. f. wissensch. Mikroskopie Bd. 29 S. 1. 1912. 
schein auftreten wie bei der Bewegung eines Geisselfadens; andererseits kann die Spirochäte während ihrer Bewegung gleichmässig hell erseheinen, oder drittens kann, besonders bei Spirochäten mit steilen Windungen, ein sehr häufiges Vorkommnis, in allen Windungen der Spirochäte ganz gleichmässig eine bestimmte Strecke hell, der übrige Teil der Windung dunkler oder völlig unsichtbar sein. Zwischen diesen verschiedenen Bildern lassen sich alle Übergänge beobachten. Si e d e n t o p f konnte nun zeigen, dass hier der wandernde Lichtschein, beziehentlich das abwechselnde Hell- und Dunkelwerden mit Sicherheit auf äussere Ursachen zurückgeführt werden muss; er fand nämlich, dass ein bei Dunkelfeldbeleuchtung abgebildetes lineares Objekt seine Helligkeit vermindern und schliesslich ganz unsichtbar werden kann, wenn es gegen die Ebene des Mikroskoptisches geneigt ist, bzw. wenn der Winkel, unter dem es gegen die Tischebene geneigt ist, eine bestimmte Grenze überschreitet. Die Länge eines Samenfadens des Frosches war im Verhältnis zu der Dicke des Präparates eine sehr grosse, so dass es nicht möglich ist, dass hier der Samenfaden als ganzes eine Neigung gegen die Tischebene des Mikroskopes annimmt, welche bereits zu Veränderungen der Helligkeit führt. Es wäre aber andererseits möglich, dass bei der immerhin rasch ablaufenden und eine erhebliche Krümmung hervorrufenden Bewegung eine kleine Strecke der Geissel sich so stark gegen die Tischebene neigen könnte, dass dadurch die Erscheinung des wandernden Lichtscheines bedingt würde.

Die Möglichkeit, die an den Geisselfäden der Froschspermatozoen bei der Bewegung wahrnehmbare Helligkeitsveränderung lediglich durch äussere, dureh die Beleuchtung bedingte Ursachen zu erklären, ist somit gegeben. Es erscheint diese Erklärung um so annehmbarer, als bei der Bewegung anderer Geisselfäden ähnliche Erscheinungen nicht beobachtet werden können, oder, wenn ein wandernder Lichtschein auftritt, dieser sich dann ohne weiteres darauf zurückführen lässt, dass die betreffenden Objekte bei ihrer Bewegung eine grössere Neigung zur Ebene des Mikroskoptisches annehmen. Hierüber seien noch kurz einige Beobacbtungen mitgeteilt.

Die Spermatozoenschwänze der Säugetiere (Maus, Ratte) sind bei Dunkelfeldbeleuchtung von denen des Frosches vor allem dadurch unterschieden, dass der Schwanz nicht einfach, sondern, abgesehen von der Pars terminalis, doppelt konturiert ist. Das Innere des Schwanzes (zwischen den beiden hellen Konturen) ist gleichmässig 
dunkel und optisch leer. An den Schwänzen der Säugetierspermatozoen lässt sich ein wandernder Lichtschein nicht beobachten. Das ist um so bemerkenswerter, als hier die Möglichkeit der Fntstehung einer stärkeren Neigung zwischen Schwanz und Ebene des Mikroskoptisches geringer ist als bei den Froschspermatozoen, denn die Spermatozoen der Säugetiere, speziell diejenigen der Maus und Ratte, sind erheblich länger als die des Frosches. Es liesse sich aber einwenden, dass auch die Geschwindigkeit der Bewegung bei den Säugetierspermatozoe山 eine viel grössere ist, und dass infolgedessen etwa bei der Bewegung auftretende Helligkeitsveränderungen der Geisselsubstanz zu raseh ablaufen, als dass sie noch subjektiv beobachtet werden könnten. Um eine so rasch ablaufende Erscheinung eventuell doch noch wahrnehmbar zu machen, wurde im mikroskopischen Laboratorium der Firma $\mathrm{C}$ arl $\mathrm{Z}$ eiss unter Leitung von Herrn Dr. Siedent opf eine kinematographische Aufnahme der Bewegung von Mäusespermatozoen angefertigt. Aber auch bei verlangsamter Projektion des Films liess sich an den Geisselfäden nirgends eine Helligkeitsveränderung beobachten, die auf eine Veränderung des Brechungsexponenten der Substanz der Geissel bezogen werden könnte.

Im Gegensatz zu den Säugetierspermatozoen liess sich an den gleichfalls sebr langen Schwänzen der Spermatozoen von Urodelen (Salamandra, Triton) der wandernde Lichtschein mit aller nur wünschenswerten Deutlichkeit beobachten. Hier ist seine Entstehung sicher so zu erklären, dass bestimmte Teile des Schwanzes bei der Bewegung eine starke Neigung zur Tischebene annehmen. Bekanntlich besitzen diese Spermatozoen eine undulierende Membran, welche in steilen Windungen um den Achsenfaden zieht, und in deren äusserem Saum der sogenannte Randfaden liegt. Die Änderungen der Helligkeit an den natürlich ebenso steilen Windungen dieses Fadens waren nun ganz dieselben wie diejenigen, welche oben an Spirochäten beschrieben wurden; auch hier kam es bis zu einem abwechselnden Aufleuchten und Dunkelwerden. - Beiläufig sei bemerkt, dass die Spermatozoen der geschwänzten Amphibien ein besonders schönes Objekt zum Studium bei Dunkelfeldbeleuchtung sind.

Ganz analoge Erscheinungen wie an Geisselzellen waren schliesslich am Flimmerepithel (Gaumen des Frosches, Kiemen der Flussmuschel) wahrnehmbar. Betrachtet man Flimmerepithelzellen, deren Cilien ihre Bewegung in der Ebene des Mikroskoptisches aus- 
124 Hans Stübel: Ultramikr. Beobachtungen an Muskel- u. Geisselzellen.

führen, so treten bei der Bewegung keine deutlichen Helligkeitsveränderungen auf. Die Erscheinung ändert sich sofort, wenn die Ebene, in welcher die Cilien schwingen, zur Tischebene geneigt ist; dann tritt je nach dem Grade dieser Neigung mit mehr oder weniger grosser Deutlichkeit das Phänomen des wandernden Lichtscheines auf; ja, es kann sogar bei sehr starker Neigung zu einem rhythmischen Verschwinden und Aufleuchten der Cilien kommen.

Es liessen sich also bis jetzt weder an glatten Muskelzellen, noch an den Myoidfäden der Protozoen, noch an Geissel- und Flimmerzellen wahrnehmbare Änderungen des Brechungsexponenten der kontraktilen Substanz bei ihrer Tätigkeit nachweisen. 